\title{
3-D Object Recognition using MEGI Model from Range Data
}

\author{
Hiroshi Matsuo \\ Akira Iwata \\ Dept. of Electrical and Computer Eng. \\ Nagoya Institute of Technology \\ Gokiso, Nagoya, 466, JAPAN \\ matsuo@mars.elcom.nitech.ac.jp
}

\begin{abstract}
Description and recognition of objects is the central considerations of research on computer vision. The key issue is how to represent 3-D objects on a machine for recognizing them. Researchers of computer vision commonly employ the EGI model, but it is not able to express concave objects. In this paper, the MEGI model and coefficient of extended spherical correlation have been proposed. The MEGI model is an extended EGI modeling which is able to represent concave objects. Extended spherical correlation is the measure for recognizing objects using the MEGI model. It has been demonstrated that this model is able to recognize 3-D objects, including concave ones, and to distinguish objects using a part of MEGI from range data.
\end{abstract}

\section{Introduction}

In order to recognize an object and to determine its attitude in space, it is necessary to have a method to represent the shape of the object. The extended Gaussian image description model (EGI) makes it easy to determine the attitude of a moving object in space [1]. It is independent of the position of the object (shift invariant). EGI provides a unique description for a convex object, though precise information is limited. That is, no two convex objects have the same EGI. In this paper, we propose a new 3-D object description called MEGI (more extended Gaussian image), which has the capability to distinguish not only convex objects, but also concave objects. This description model is a shift-invariant model which consists of a set of position vectors of surfaces originating from the center of a 3-D object and normal vectors of the surfaces. We also propose a matching scale function called extended spherical correlation, which is rotational and scale invariant, together with a calculation algorithm for this scale function.

\section{More extended Gaussian image (MEGI) model}

Horn and Smith[1] have proposed the extended Gaussian image model as a description model for representing and analyzing 3-D objects. However, this representation does not include information on connections of each surface, and therefore it cannot represent concave objects, which commonly exist in the usual 3-D object recognition.

The problems described above are caused by information loss for surface position. To solve these problems we proposed a new 3-D shape description method called more extended Gaussian image (MEGI), which includes information on surface positions, so it can describe not only convex but also concave objects.

The MEGI model consists of a set of position vectors $X_{i}$ for surfaces originating from an object center and their normal vectors $p_{i}$. Each length of a normal vector also corresponds with surface area, as in the extended Gaussian image. This model is also shiftinvariant since it is expressed by an object-oriented coordinate.

Let $\mathbf{M}$ be a vector set which describes vectors of the MEGI element as follows:

$$
\begin{aligned}
\mathbf{M}= & \left\{\left(\mathbf{X}_{i}, \mathbf{p}_{i}\right) \mid i \in\{0,1, \cdots, m-1\}\right\} \\
& \mathbf{X}_{i}, \mathbf{p}_{i} \in \mathbf{R}^{3} .
\end{aligned}
$$

$m$ indicates number of MEGI elements.

\section{Extended Spherical Correlation}

\subsection{Formulation of spherical correlation}

Let $\mathbf{X}$ and $\mathbf{Y}$ be sets of $n$-dimensional unit vectors and let the elements be $\mathbf{X}=\left\{\mathbf{X}_{0}, \mathbf{X}_{1}, \ldots, \mathbf{X}_{m-1}\right\}$, $\mathbf{Y}=\left\{\mathbf{Y}_{0}, \mathbf{Y}_{1}, \ldots, \mathbf{Y}_{m-1}\right\} \quad\left(\left\|\mathbf{X}_{i}\right\|=\left\|\mathbf{Y}_{i}\right\|=1\right)$. 
Fisher and Lee[2] proposed the spherical correlation coefficients which are defined as follows:

$$
\rho=\frac{\operatorname{det}\left\{\sum_{i} \mathbf{X}_{i} \mathbf{Y}_{i}^{\prime}\right\}}{\sqrt{\operatorname{det}\left\{\sum_{i} \mathbf{X}_{i} \mathbf{X}_{i}^{\prime}\right\} \operatorname{det}\left\{\sum_{i} \mathbf{Y}_{i} \mathbf{Y}_{i}^{\prime}\right\}}}
$$

\subsection{Definition of the extended spherical correlation}

We propose a new coefficient for recognizing 3-D objects with the MEGI model, which is an extension of the spherical correlation coefficients proposed by Fisher and Lee. The definition is as follows.

$$
\begin{aligned}
E C & =m_{1}{ }^{\alpha} * m_{2}{ }^{\beta} \\
m_{1} & =\frac{1}{2}\left(\frac{\operatorname{det}\left\{\sum_{i} \mathbf{p}_{i} \mathbf{q}_{i}^{\prime}\right\}}{\sqrt{\operatorname{det}\left\{\sum_{i} \mathbf{p}_{i} \mathbf{p}_{i}^{\prime}\right\} \operatorname{det}\left\{\sum_{i} \mathbf{q}_{i} \mathbf{q}_{i}^{\prime}\right\}}}+1\right) \\
m_{2} & =\frac{1}{(\gamma|\log (S)|+1) d_{q}} \\
\sum_{i}\left(1-\frac{\left|\sum_{j \in d(i)}\left\|\mathbf{p}_{j}\right\|-\frac{\left\|\mathbf{q}_{i}\right\|}{S}\right|}{\sum_{j \in d(i)}\left\|\mathbf{p}_{j}\right\|+\frac{\left\|\mathbf{q}_{i}\right\|}{S}}\right) & \frac{\left\|\mathbf{q}_{0}\right\|}{\sum_{j \in d(0)}\left\|\mathbf{p}_{j}\right\|}
\end{aligned}
$$

where $\mathbf{p}_{i}$ and $\mathbf{q}_{i}$ are the normal vectors for object $A$ and object $\mathrm{B}$ which calculate the correlations, $d_{q}$ denotes the number of vectors $\mathbf{q}, \alpha, \beta$ and $\gamma$ are constant values, " 1 " in the equations denotes the transpose of the matrix, and $d(i)$ denotes the set of the vector number of $\mathbf{p}$ which corresponds to $\mathbf{q}_{i}$.

Equation (4) is a function having a range $(0 \leq$ $\left.m_{1} \leq 1\right)$ which is derived from the spherical correlation coefficients by Fisher and Lee. Equation (5) describes the difference in volume between the two objects. $\alpha, \beta$ and $\gamma$ are the constant values whose parameters control the behavior of the coefficient. The parameter $\alpha$ sets the influence of the original spherical correlation, the parameter $\beta$ controls the influence of the difference between surface areas, and the parameter $\gamma$ controls the influence of the volume difference between objects for the extended spherical correlation.

The value calculated by equation (4) is rotational invariant since Fisher's spherical correlation coefficients are rotational invariant. $m_{2}$ derived from equation $(5)$ is a scalar value. Therefore the extended spherical correlation defined by equation (3) is also rotational invariant. Hence using the MEGI model proposed in $\mathbf{2}$ and the extended spherical correction, we can estimate the matching score which is shift and rotational invariant.

\section{Matching Algorithm Using MEGI}

\subsection{Correspondence determination func- tion}

Finding a matching pair of objects is done by finding the maximum EC defined by equation (3) between objects. In order to calculate the EC which was defined in 3.2, the correspondence of each MEGI element (a set of position vectors and normal vectors) must be known. But this correspondence is unknown for general 3-D objects. Therefore a correspondence procedure for MEGI elements of two objects must be performed in some way. We propose the correspondence determination function (CF) in equations (7) - (10) for determining the correspondence between MEGI elements of two objects.

$$
\begin{aligned}
C F & =n_{1}{ }^{a} * n_{2}{ }^{b} * n_{3}{ }^{c} \\
n_{1} & =\frac{1}{2}\left(\frac{\overline{\mathbf{X}}_{i} \mathbf{Y}_{j}}{\left\|\overline{\mathbf{X}}_{i}\right\|\left\|\mathbf{Y}_{j}\right\|}+1\right) \\
n_{2} & =1-\frac{\left\|\overline{\mathbf{X}}_{i}\right\|-\left\|\mathbf{Y}_{j}\right\| \|}{\max _{k}\left\|\overline{\mathbf{X}}_{k}\right\|+\max _{k}\left\|\mathbf{Y}_{k}\right\|} \\
n_{3} & =\frac{1}{2}\left(\frac{\mathbf{p}_{i}^{\prime} \mathbf{q}_{j}}{\left\|\mathbf{p}_{i}\right\|\left\|\mathbf{q}_{j}\right\|}+1\right)
\end{aligned}
$$

where $\overline{\mathbf{X}}_{i}$ is the position vector after a projective transformation is performed, $\mathbf{Y}_{j}$ is the position vector of the object $\mathrm{B}, \mathbf{p}_{i}$ and $\mathbf{q}_{j}$ are the normal vectors which correspond to $\overline{\mathbf{X}}_{i}$ and $\mathbf{Y}_{j}$, respectively. The algorithm for projective transformation is described in 4.3. $a, b$ and $c$ are constants.

In determining the correspondence of the MEGI elements, a standard surface should be selected. Then each position vector of the other surface is expressed as a relative vector from the center of the selected standard surface. Hence the correspondence determination function is not influenced by movement of the center of the object. Performing these projective transformations on the set of MEGI elements of object $\mathrm{A}$, it is possible to obtain a flexible matching against rotation, magnification and reduction and distortion of the object shape.

Equations (8) and (9) are similarity factors of direction and magnitude derived from each corresponding position vector selected from two objects, respectively. Equation (10) is a similarity factor of direction derived from the normal vector corresponding to the position 
vector of equations (8) and (9). $a, b$ and $c$ are constant values which control the sensitivity of the difference between the direction of position vectors, magnitude of position vectors, and the direction of the normal vectors for the correspondence determination function $(\mathrm{CF})$.

In the corresponding procedure, object $\mathrm{A}$ is used as a standard object. Therefore, if the number of MEGI elements of object $\mathrm{A}$ is greater than the number of MEGI elements of object B, more than two elements of object B correspond to one MEGI elements of object A. On the other hand, if the number of MEGI elements of object $\mathrm{A}$ is less than the number of MEGI elements of object B, some element of object B which cannot correspond to MEGI elements of object A exists. Using these corresponding methods, partial matching, such as where object $A$ is a part of object $B$, can be performed.

\subsection{In the case where the spherical corre- lation cannot be defined}

The conditions of calculating $m_{1}$ which are expressed as equation (3) are the following. First, the number of MEGI elements is greater than four. Second, at least one set of position vectors within all the combinations for selecting three vectors from the MEGI element of the object is independent; in other words, there are at least three position vector sets which have different directions. This condition is satisfied easily for general 3-D objects, but it cannot be satisfied for data which consists of a range data observed from one side of the object. In this case, $m_{1}^{\prime}$, which is defined as follows,

$$
\begin{aligned}
m_{1}^{\prime}= & \frac{1}{m(m-1)} \\
& \sum_{i, j, i \neq j}\left(1-\frac{1}{2}\left|\frac{\mathbf{p}_{i} \cdot \mathbf{p}_{j}}{\left\|\mathbf{p}_{i}\right\|\left\|\mathbf{p}_{j}\right\|}-\frac{\mathbf{q}_{i} \cdot \mathbf{q}_{j}}{\left\|\mathbf{q}_{i}\right\|\left\|\mathbf{q}_{j}\right\|}\right|\right)
\end{aligned}
$$

is used instead of $m_{1}$ in equation (4). It represents the difference of each corresponding angle between two normal vectors which are selected from normal vector sets of object $\mathrm{A}$ and object $\mathrm{B}$, respectively. The range of $m_{1}^{\prime}$ in this equation is $-1 \leq m_{1}^{\prime} \leq 1$, and $m_{1}^{\prime}$ is rotational-invariant, as in equation (4).

\subsection{Matching Algorithm}

The matching process for each MEGI elements is performed using the following algorithm. Case 2 is the special stage when the spherical correlation cannot be defined.

$\underline{\text { Case } 1}$
Step 1 If one of two objects has less than three MEGI elements, go to Case 2

Step 2 A standard surface is selected from object A as that with the largest surface area. Next, the most orthogonal three vectors which are translated to relative vectors originating from the center of the standard surface are selected, which vector set we call "the basis of object A." If no independent vector set can be obtained for any surface, then go to Case 2.

Step 3 The standard surface and some sets of surfaces which have position vectors similar to the basis of object A are selected from object B. We call these selected vector sets " the basis of object B." If an independent basis set cannot be obtained, then go to Case 2.

Step 4 Projective transformation is performed for each MEGI element of object $A$, using the basis of object $B$.

Step 5 The element of object B which takes the largest value of correspondence determination function corresponds to the element of object A after projective transformation.

Step 6 The extended spherical correlation is calculated (the standard surface selected at Step 3 denotes vector number 0 in equation (6). If all normal vectors take the same direction, $m_{1}^{\prime}$ defined by equation (11) is used instead of $m_{1}$ in equation (4).

Step 7 The extended spherical correlation for all sets of standard surfaces and the basis selected at Step 3 are calculated. The largest value of the calculated extended spherical correlation is defined as the correlation between two objects.

\section{$\underline{\text { Case } 2}$}

Step 1 All possible corresponding sets are calculated between MEGI elements of two objects.

Step 2 The extended spherical correlation for all sets is calculated using $m_{1}^{\prime}$ in equation (11) instead of $m_{1}$ defined by equation (4).

Step 3 The extended spherical correlation for all sets selected at Step 1, is calculated. The largest value of the extended spherical correlation is defined as the correlation between two objects. 
Table 1: The matching result with real range data

\begin{tabular}{|c|c|l|l|l|l|l|}
\hline \multicolumn{2}{|c|}{} & \multicolumn{5}{|c|}{ MEGI data from real range data } \\
\cline { 3 - 7 } \multicolumn{2}{|c|}{} & object a & object b & object c & object d & object e \\
\hline \multirow{2}{*}{ MEGI } & object a & $0.92(1)$ & $0.37(3)$ & $0.65(5)$ & $0.17(5)$ & $0.20(5)$ \\
\cline { 2 - 7 } data & object b & $0.85(3)$ & $0.86(1)$ & $0.66(4)$ & $0.36(2)$ & $0.39(4)$ \\
\cline { 2 - 7 } from & object c & $0.86(2)$ & $0.23(4)$ & $0.88(1)$ & $0.18(4)$ & $0.50(2)$ \\
\cline { 2 - 7 } CAD & object d & $0.83(4)$ & $0.49(2)$ & $0.71(3)$ & $0.85(1)$ & $0.43(3)$ \\
\cline { 2 - 7 } model & object e & $0.72(5)$ & $0.23(4)$ & $0.73(2)$ & $0.23(3)$ & $0.80(1)$ \\
\hline
\end{tabular}

\section{$5 \quad$ Experimental Results}

This matching algorithm using the MEGI model has been implemented, and we show a example of 3$\mathrm{D}$ object recognition using real range data. In the experiments, coefficients $a, b$ and $c$ in equation (7) are set at 1.0. Sixty-four bases of objects described at Step 3 in $\mathbf{4 . 3}$ are selected. The experiments involving real range data[3] of five objects were measured. The five acquired range data shown in Figure 1. has much occluded surface. And we also constructed five 3-D shape models (CAD model) respectively.

Table 1 shows the matching correlation using the extended spherical correlation between acquired range data taken from one side of an object and constructed it's 3-D models. From this experimental result, it is possible to recognize the object from the range data using the extended spherical correlation calculated from MEGI, even if there are some occluded surface in the measured range data.

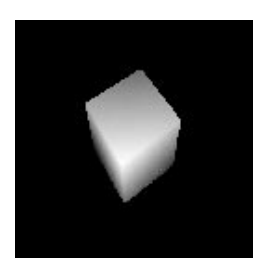

(a)

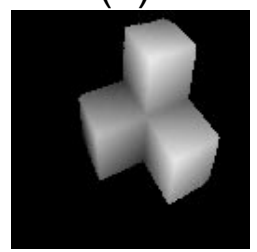

(d)

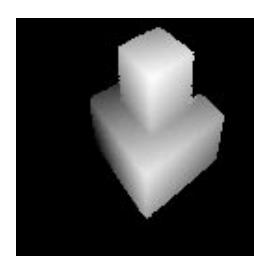

(b)

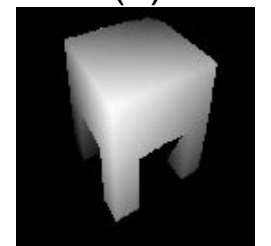

(e)

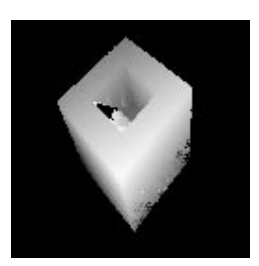

(c)

\section{Conclusions}

A description method for recognizing 3-D objects including convex and concave shapes has been developed. This description is an extended description from extended Gaussian image. A recognition method using the extended spherical correlation is also developed in this paper. Furthermore, a matching algorithm for MEGI and an extended spherical correlation are also proposed.

MEGI is a model which has position information for each surface in addition to normal vector information on the object. Adding this extension, it becomes possible to recognize a 3 -D object including convex and concave shapes which is impossible to recognize using EGI. Presently, experiments using complex shapes obtained from real range sensors with noise being conducted by our group.

\section{ACKNOWLEDGMENT}

The authors are grateful to Associate Professor Y. Sato of the Department of Electrical and Computer Engineering, Nagoya Institute of Technology, who supplied the range date using section 5 , and to former and current students K. Tsuchiya, T. Okamoto and J. Funabashi. This work was supported by Artificial Intelligence Research Promotion Foundation in Japan.

\section{References}

[1] B. Horn, "Extended Gaussian Images", Proc. of IEEE, vol.72, no.12, pp.1671-1686, Dec. 1984

[2] N. Fisher, A. Lee, "Correlation Coefficients for Random Variables on a Unit Sphere or Hypersphere", Biometrica, vol.73, pp.159-164 (1986)

[3] Y. Sato, M. Otsuki, "Three-dimensional Shape Reconstruction by Active Rangefinder", Proc. IEEE CVPR, pp.142-147, 1993

Figure 1: Real range data 